\title{
Exploring the Utility of Artificial Intelligence During COVID-19 in Dermatology Practice
}

\author{
Geeta Ahuja, BS; Sarine Tahmazian, BS; Sofiat Atoba, BS; Kamaria Nelson, MD, MHS
}

\section{PRACTICE POINTS}

- Dermatologists should amass pictures of dermatologic conditions in skin of color to contribute to growing awareness and knowledge of presentation of disease in this population.

- Dermatologists should use artificial intelligence as a tool for delivering more efficient and beneficial patient care.

W ith the need to adapt to the given challenges associated with COVID-19, artificial intelligence (AI) serves as a potential tool in providing access to medical-based diagnosis in a novel way. Artificial intelligence is defined as intelligence harnessed by machines that have the ability to perform what is called cognitive thinking and to mimic the problem-solving abilities of the human mind. Virtual AI in dermatology entails neural network-based guidance that includes developing algorithms to detect skin pathology through photographs. ${ }^{1}$ To use AI in dermatology, recognition of visual patterns must be established to give diagnoses. These neural networks have been used to classify skin diseases, including cancer, actinic keratosis, and warts. ${ }^{2}$

\section{Al for Skin Cancer}

The use of AI to classify melanoma and nonmelanoma skin cancer has been studied extensively, including the following 2 research projects.

Convolutional Neural Network-In 2017, Stanford University published a study in which a deep-learning algorithm known as a convolutional neural network was used to classify skin lesions. ${ }^{3}$ The network was trained using a dataset of 129,450 clinical images of 2032 diseases. Its performance was compared to that of 21 board-certified dermatologists on biopsy-proven clinical images with 2 classifications of cases: (1) keratinocyte carcinoma as opposed to benign seborrheic keratosis and (2) malignant melanoma as opposed to benign nevi-the first representing the most common skin cancers, and the second, the deadliest skin cancers. The study showed that the machine could accurately identify and classify skin cancers compared to the work of board-certified dermatologists. The study did not include demographic information, which limits its external validity. ${ }^{3}$

Dermoscopic Image Classification-A 2019 study by Brinker and colleagues ${ }^{4}$ showed the superiority of automated dermoscopic melanoma image classifications compared to the work of board-certified dermatologists. For the study, 804 biopsy-proven images of melanoma and nevi (1:1 ratio) were randomly presented to dermatologists for their evaluation and recommended treatment (yielding 19,296 recommendations). The dermatologists classified the lesions with a sensitivity of $67.2 \%$ and specificity of $62.2 \%$; the trained convolutional neural network attained both higher sensitivity (82.3\%) and higher specificity (77.9\%). ${ }^{4}$

\section{Smartphone Diagnosis of Melanoma}

An application of AI has been to use smartphone apps for the diagnosis of melanoma. The most utilized and novel algorithm-based smartphone app that assesses skin lesions for malignancy characteristics is SkinVision. With a simple download from Apple's App Store, this technology allows a person to check their skin spots by taking a photograph and receiving algorithmic risk-assessment feedback. This inexpensive software ( $\$ 51.78$ a year) also allows a patient's physician to assess the photograph and then validate their assessment by comparing it with the algorithmic analysis that the program provides. ${ }^{5}$

A review of SkinVision conducted by Thissen and colleagues $^{6}$ found that, in a hypothetical population of

Ms. Ahuja, Ms. Tahmazian, and Ms. Atoba are from Howard University College of Medicine, Washington, DC. Dr. Nelson is from the Department of Dermatology, George Washington University, Washington, DC.

The authors report no conflict of interest.

Correspondence: Geeta Ahuja, BS, 13533 Ann Grigsby Circle, Centreville, VA 20120 (geeta.ahuja@bison.howard.edu). doi:10.12788/cutis.0318 
1000 adults of whom 3\% actually had melanoma, 4 of those 30 people would not have been flagged as at "high risk" by SkinVision. There also was a high false-positive rate with the app, with more than 200 people flagged as at high risk. The analysis pegged SkinVision as having a sensitivity of $88 \%$ and specificity of $79 \%{ }^{6}$

In summary, systematic review of diagnostic accuracy has shown that, although there is accuracy in AI analyses, it should be used only as a guide for health care advice due to variability in algorithm performance. ${ }^{7}$

\section{Utility of Al in Telehealth}

Artificial intelligence algorithms could be created to ensure telehealth image accuracy, stratify risk, and track patient progress. With teledermatology visits on the rise during the COVID-19 pandemic, AI algorithms could ensure that photographs of appropriate quality are taken. Also, patients could be organized by risk factors with such algorithms, allowing physicians to save time on triage and stratification. Algorithms also could be used to track a telehealth patient's treatment and progress. ${ }^{8}$

Furthermore, there is a need for an algorithm that has the ability to detect, quantify, and monitor changes in dermatologic conditions using images that patients have uploaded. This capability will lead to creation of a standardized quantification scale that will allow physicians to virtually track the progression of visible skin pathologies.

\section{Hazards of Racial Bias in Al}

Artificial intelligence is limited by racial disparity bias seen in computerized medicine. For years, the majority of dermatology research, especially in skin cancer, has been conducted on fairer-skinned populations. This bias has existed at the expense of darker-skinned patients, whose skin conditions and symptoms present differently, ${ }^{9}$ and reflects directly in available data sets that can be used to develop $\mathrm{AI}$ algorithms. Because these data are inadequate to the task, AI might misdiagnose skin cancer in people of color or miss an existing condition entirely..$^{10}$ Consequently, the higher rate of skin cancer mortality that is reported in people of color is likely to persist with the rise of $\mathrm{AI}$ in dermatology. ${ }^{11} \mathrm{~A}$ more representative database of imaged skin lesions needs to be utilized to create a diversely representative and applicable data set for $\mathrm{AI}$ algorithms. ${ }^{12}$

\section{Benefits of Conversational Agents}

Another method by which AI could be incorporated into dermatology is through what is known as a conversational agent (CA) - AI software that engages in a dialogue with users by interpreting their voice and replying to them through text, image, or voice. ${ }^{13}$ Conversational agents facilitate remote patient management, allow clinicians to focus on other functions, and aid in data collection. ${ }^{14} \mathrm{~A}$ 2014 study showed that patients were significantly more likely to disclose history and emotions when informed they were interacting with a CA than with a human clinician $(P=.007) .{ }^{15}$ Such benefits could be invaluable in dermatology, where emotions and patient perceptions of skin conditions play into the treatment process.

However, some evidence showed that CAs cannot respond to patients' statements in all circumstances. ${ }^{16}$ It also is unclear how well CAs recognize nuanced statements that might signal potential harm. This fits into the greater theme of a major problem with AI: the lack of a reliable response in all circumstances. ${ }^{13}$

\section{Final Thoughts}

The practical implementations of $\mathrm{AI}$ in dermatology are still being explored. Given the uncertainty surrounding the COVID-19 pandemic and the future of patient care, AI might serve as an important asset in assisting with the diagnosis and treatment of dermatologic conditions, physician productivity, and patient monitoring.

\section{REFERENCES}

1. Amisha, Malik $P$, Pathania $M$, et al. Overview of artificial intelligence in medicine. J Family Med Prim Care. 2019;8:2328-2331. doi:10.4103/jfmpc.jfmpc_440_19

2. Han SS, Kim MS, Lim W, et al. Classification of the clinical images for benign and malignant cutaneous tumors using a deep learning algorithm. J Invest Dermatol. 2018;138:1529-1538. doi:10.1016/j.jid.2018.01.028

3. Esteva A, Kuprel B, Novoa RA, et al. Dermatologist-level classification of skin cancer with deep neural networks. Nature. 2017;542:115-118. doi:10.1038/nature21056

Brinker TJ, Hekler A, Enk AH, et al. Deep neural networks are superior to dermatologists in melanoma image classification. Eur J Cancer. 2019;119:11-17. doi:10.1016/j.ejca.2019.05.023

5. Regulated medical device for detecting skin cancer. SkinVision website. Accessed July 23, 2021. https://www.skinvision.com/hcp/

6. Thissen M, Udrea A, Hacking $M$, et al. mHealth app for risk assessment of pigmented and nonpigmented skin lesions - a study on sensitivity and specificity in detecting malignancy. Telemed J E Health. 2017;23:948-954. doi:10.1089/tmj.2016.0259

7. Freeman K, Dinnes J, Chuchu N, et al. Algorithm based smartphone apps to assess risk of skin cancer in adults: systematic review of diagnostic accuracy studies. BMJ. 2020;368:m127. doi:10.1136/bmj.m127

8. Puri P, Comfere N, Pittelkow MR, et al. COVID-19: an opportunity to build dermatology's digital future. Dermatol Ther. 2020;33:e14149. doi:10.1111/dth.14149

9. Buster KJ, Stevens EI, Elmets CA. Dermatologic health disparities. Dermatol Clin. 2012;30:53-59,viii. doi:10.1016/j.det.2011.08.002

10. Adamson AS, Smith A. Machine learning and health care disparities in dermatology. JAMA Dermatol. 2018;154:1247-1248. doi:10.1001 /jamadermatol.2018.2348

11. Agbai ON, Buster K, Sanchez M, et al. Skin cancer and photoprotection in people of color: a review and recommendations for physicians and the public. J Am Acad Dermatol. 2014;70:748-762. doi:S0190-9622(13)01296-6

12. Alabdulkareem A. Artificial intelligence and dermatologists: friends or foes? J Dermatol Dermatolog Surg. 2019;23:57-60. doi:10.4103/jdds.jdds_19_19

13. McGreevey JD 3rd, Hanson CW 3rd, Koppel R. Clinical, legal, and ethical aspects of artificial intelligence-assisted conversational agents in health care. JAMA. 2020;324:552-553. doi:10.1001/jama.2020.2724

14. Piau A, Crissey R, Brechemier D, et al. A smartphone chatbot application to optimize monitoring of older patients with cancer. Int J Med Inform. 2019;128:18-23. doi:10.1016/j.jmedinf.2019.05.013

15. Lucas GM, Gratch J, King A, et al. It's only a computer: virtual humans increase willingness to disclose. Comput Human Behav. 2014;37:94-100. https://doi.org/10.1016/j.chb.2014.04.043

16. Miner AS, Milstein A, Schueller S, et al. Smartphone-based conversational agents and responses to questions about mental health, interpersonal violence, and physical health. JAMA Intern Med. 2016; 176:619-625. doi:10.1001/jamainternmed.2016.0400 